\title{
A size and age dependence study of shower front curvature with GRAPES-3 array.
}

\author{
V.B. Jhansi ${ }^{* a b}$ S. Ahmad ${ }^{a c}$, A. Chandra ${ }^{a b}$, S.R. Dugad ${ }^{a b}$, S.K. Gupta ${ }^{a b}$, B. \\ Hariharan $^{a b}$, Y. Hayashi ${ }^{a d}$, P. Jagadeesan ${ }^{a b}$, A. Jain ${ }^{a b}$, S. Kawakami ${ }^{a d}$, H. Kojima ${ }^{a e}$, \\ P.K. Mohanty ${ }^{a b}$, S.D. Morris ${ }^{a b}$, P.K.Nayak ${ }^{a b}$, A. Oshima ${ }^{a f}$, B.S. Rao ${ }^{a b}$, L.V. Reddy ${ }^{a b}$, S. \\ Shibata $^{a f}$, M.Zuberi $^{a b}$ \\ ${ }^{a}$ The GRAPES-3 Experiment, Cosmic Ray Laboratory, Raj Bhavan, Ooty 643001, India \\ ${ }^{b}$ Tata Institute of Fundamental Research, Mumbai 400005, India \\ ${ }^{c}$ Aligarh Muslim Univeristy, Aligarh 202002, India \\ ${ }^{d}$ Graduate School of Science, Osaka City University, Osaka 558-8585, Japan \\ ${ }^{e}$ Faculty of Engineering, Aichi Insitute of Technology, Toyota City, Aichi 470-0392, Japan \\ ${ }^{f}$ College of Engineering, Chubu University, Kasugai, Aichi 487-8501, Japan \\ E-mail: jhansi1990@gmail.com
}

\begin{abstract}
Extensive air showers spread laterally mainly when electrons undergo multiple coulumb scattering in the atmosphere. The low energy secondaries and tertiaries will scatter further and will end up farther from the shower core. The particles which are scattered at larger angles during the shower development have higher path lengths and they arrive at the observational level slightly later. Moreover, the particles farther from the core, being lower in energy travel slower giving rise to additional time delays. The time delays, so produced, give rise to a shower front which is non planar in shape. The shape of the shower front has been determined to be conical by various air shower experiments. Here, we present the study of shower front curvature with the data from GRAPES-3 array. We observed that the shower front can be well approximated with a conical shape. However, our detail study has shown that the slope of the conical front has a strong dependence on both shower size and age. The correction of these dependence has led to an improvement in the angular resolution by a factor of two. This would provide efficient observation of multi-TeV gamma ray sources with GRAPES-3.
\end{abstract}

36th International Cosmic Ray Conference -ICRC2019-

July 24th - August 1st, 2019

Madison, WI, U.S.A.

* Speaker. 


\section{GRAPES-3 Array}

The GRAPES-3 EAS array is located at Ooty $\left(11.4^{0} \mathrm{~N}\right.$ latitude, $76.7^{0} \mathrm{E}$ longitude $)$ at an atlitude of $2200 \mathrm{~m}$ above the mean sea level. In this array, about 400 plastic scintillator detectors are deployed over a total area of $25000 \mathrm{~m}^{2}$. The detectors are arranged in concentric hexagonal rings such that the seperation between the adjacent detectors is only $\sim 8 \mathrm{~m}$. Each scintillator detector contains four scintillator slabs each of area $50 \times 50 \mathrm{~m}^{2}$. The active area occupied by the array is $\sim 2 \%$, making it one of the densest arrays of the world. In experiments, such as KASCADE, GAMMA, EAS-TOP etc, the active area is about $\sim 1 \%$. It is expected that a larger active area would result in an improved angular resolution[7].

Each scintillator detector is equipped with a photomultiplier tube (ET9807B). On the arrival of an extensive air shower (EAS), the charged particles deposit a certain amount of energy in the scintilltor, resulting in the emission of photons. These photons are collected by the PMT and converted into an electrical pulse. Typically, the electrical signal is characterized by a rise time of $\sim 3 \mathrm{~ns}$ and an amplitude of $\sim$ few MeV. A part of the signal is fed into a 15-bit charge integrating analog-to-digital converters (ADC). The ADC digitizes and stores the integrated charge of the signal. The integrated charge of the signal is a measure of the particle number recorded in each detector during the passage of an EAS. The remaining part of the signal is used to obtain the arrival time information of the PMT signal. The arrival time of the signal is digitized using time-to-digital converters (TDC) with a time resolution of $195 \mathrm{ps}$. The digitization process in the ADC and TDC, begins only on the arrival of the Level-0 trigger. The Level-0 trigger is employed to achieve a low energy threshold for the registration of EAS. In order to eliminate large showers of which the cores are incident outside the array, a second level of trigger is implemented namely the Level-1 trigger. The steps involved in the generation of Level-0 and Level-1 are discussed elsewhere[4].

\section{Determination of shower slope}

An extensive air shower spreads laterally due to transverse momenta imparted to particles during strong interactions, and due to multiple coulumb scattering from the electric fields of air nuclei. The particles which are scattered at larger angles during shower development have to traverse larger path lengths and they arrive at the observational level slightly later[7][2], giving rise to a showerfront which is non planar in shape[6][1]. In order to determine the shape of the shower front, it is required to determine the approximate direction of the shower by approximating the shower front as a plane. The shower plane, which is perpendicular to the direction of the primary particle (also called the shower axis) is determined by fitting the arrival times of particles with a plane.

The plane front is represented by the equation

$$
\mathrm{lx}+\mathrm{my}+\mathrm{nz}-\mathrm{ct}=0
$$

where $\mathrm{l}=\sin (\theta) \cos (\phi), \mathrm{m}=\cos (\theta) \sin (\phi)$ and $\mathrm{n}=\cos (\theta)$ are the direction cosines of the shower axis, $\theta$ and $\phi$ are the zenith angles and azimuth angles, $\mathrm{x}, \mathrm{y}$ and $\mathrm{z}$ are the cartesian co-ordinates, $\mathrm{c}$ is the velocity of light and $t$ is the time of arrival of the front at the point relative to some arbitrary time. The fitting is carried out by minimizing the quantity $\psi^{2}$ defined as 


$$
\psi^{2}=\Sigma_{\mathrm{i}}\left(\mathrm{lx}_{\mathrm{i}}+\mathrm{my}_{\mathrm{i}}+\mathrm{nz}_{\mathrm{i}}-\mathrm{c}\left(\mathrm{t}_{\mathrm{i}}-\mathrm{t}_{0}\right)\right)^{2}
$$

where $t_{i}$ is the arrival time at the $i^{t h}$ detector with co-ordinates $x_{i}, y_{i}$ and $z_{i}$ are the cartesian coordinates and the velocity of light and $t_{0}$ is the reference time[8]. The core location $(\mathrm{X}, \mathrm{Y})$, shower size $\mathrm{N}_{\mathrm{e}}$ and age parameter $\mathrm{s}$ of an extensive air shower is estimated by fitting the lateral distribution of charged particles with NKG function $\Delta\left(\mathrm{N}_{\mathrm{e}}, \mathrm{r}\right)$ using Maximum Likelihood Method .

$$
\left.\Delta\left(\mathrm{N}_{\mathrm{e}}, \mathrm{r}\right)=\frac{\mathrm{N}_{\mathrm{e}}}{2 \pi \mathrm{r}_{0}^{2}} \frac{\Gamma(4.5-\mathrm{s})}{\Gamma(\mathrm{s}) \Gamma(4.5-2 \mathrm{~s})}\left(\frac{\mathrm{r}}{\mathrm{r}_{0}}\right)^{(\mathrm{s}-2)}\left(1+\frac{\mathrm{r}}{\mathrm{r}_{0}}\right)^{\mathrm{s}-4.5}\right]
$$

where $\Delta\left(\mathrm{N}_{\mathrm{e}}, \mathrm{r}\right)$ is the density of electrons per $\mathrm{m}^{2}$ at a distance $\mathrm{r}$ in a plane perpendicular to the shower axis in a shower with total number of electrons $\mathrm{N}_{\mathrm{e}}, \Gamma$ is the gamma function, $s$ is the age parameter and $r_{0}=103 \mathrm{~m}$ is the Moliere unit which has been calculated for the atmospheric pressure of Ooty[8][5].

For each shower, the time residual $\mathrm{t}_{\text {res }}^{\mathrm{i}}$ of each detector with respect to planar fit is calculated as time difference between the arrival times as determined from the planar fit $\left(\mathrm{t}_{\mathrm{i}}^{\mathrm{p}}\right)$ and the measured arrival times of particles $t_{i}$ (also denoted as $t_{i}^{m}$ )

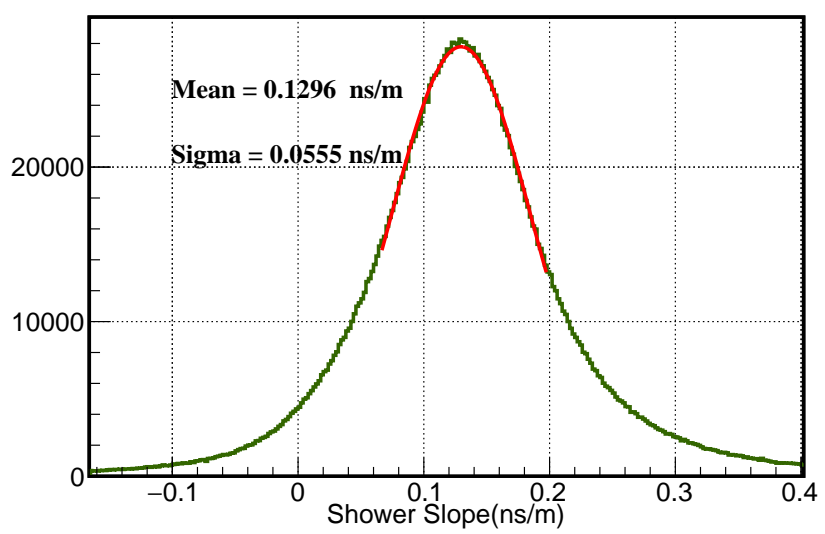

Figure 1: The final shower slope distribution after removing outliers

$$
\begin{gathered}
\mathrm{t}_{\mathrm{i}}^{\mathrm{p}}=\mathrm{t}_{0}-\frac{1 \mathrm{x}_{\mathrm{i}}+\mathrm{my}_{\mathrm{i}}+\mathrm{nz}_{\mathrm{i}}}{\mathrm{c}} \\
\mathrm{t}_{\mathrm{i}}^{\text {res }}=\mathrm{t}_{\mathrm{i}}^{\mathrm{p}}-\mathrm{t}_{\mathrm{i}}^{\mathrm{m}}
\end{gathered}
$$

Furthermore, the distance of each detector $r_{i}$ from the shower core in the plane perpendicular to the shower axis is calculated as $r_{i}=\sqrt{\left.\left(x_{i}-X\right)\left(x_{i}-X\right)+\left(y_{i}-Y\right)\left(y_{i}-Y\right)-D^{2}\right)}$ where $\mathrm{D}=\left(\mathrm{x}_{\mathrm{i}}-\mathrm{X}\right) \sin (\theta) \cos (\phi)+\left(\mathrm{y}_{\mathrm{i}}-\mathrm{Y}\right) \sin (\theta) \sin (\phi), \mathrm{X}$ and $\mathrm{Y}$ are the positions of shower core [5].

Since the shielding coverage of muon detector extends only upto $45^{\circ}$, the showers for further analysis have been selected upto maximum zenith angle of $40^{\circ}$. A further cut was imposed on the location of the shower core to be within $30 \mathrm{~m}$ from the centre of the array. The time residuals of extensive air showers is observed to be increasing linearly with perpendicular distance from the 
shower core. A linear fit to this observed variation yields the slope of the EAS. The distribution is then fitted with a Gaussian function thereby obtaining the mean value of shower slope to be $0.141 \mathrm{~ns} / \mathrm{m}$. The shower slopes were recalculated by removing outliers iteratively, until their mean value converged to $0.129 \mathrm{~ns} / \mathrm{m}$ in the third iteration. The outliers were filtered out using a $2 \sigma$ cut, resulting only in the elimination of $13.6 \%$ of the detectors per shower. The distribution of shower slopes are shown in Figure 1.

\section{Dependence of shower slope on shower size and age}

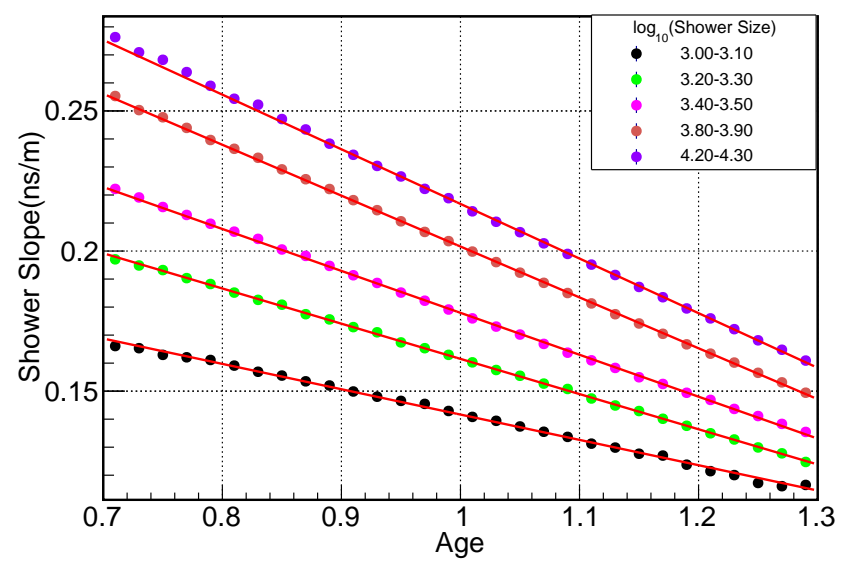

Figure 2: Variation of shower slope with age and shower size

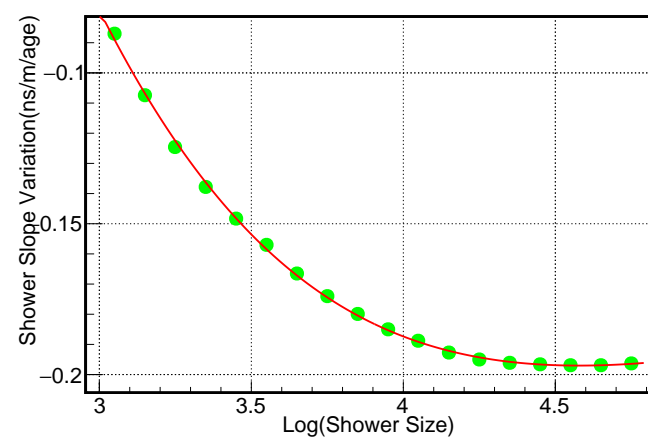

(a) Variation of $m_{\text {size }}$ with shower size

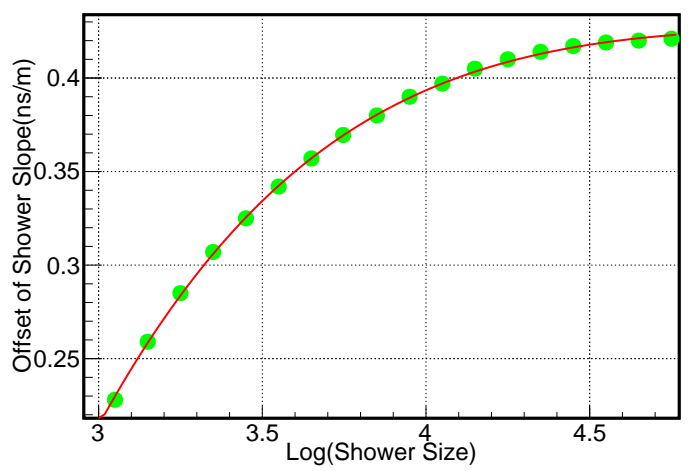

(b) Variation of $\mathrm{c}_{\text {size }}$ with shower size

Figure 3

To probe the dependence of shower slope on shower size and age, a total number of about 1 billion showers, collected over a period of 365 days from January 12014 to December 312014 were categorized into 20 shower size bins each of binwidth 0.1 in the logarithmic scale. The variation of shower slopes with respect to age and shower size are shown in the Fig. 2 . There are three major inferences that can be drawn from this figure. 
Table 1: The functional depedence of shower slope on age and shower size

\begin{tabular}{|c|c|c|}
\hline Variable & Functional Form & Fitted Parameters \\
\hline $\mathrm{m}_{\text {size }}$ & $a_{1} \exp \left(-b_{1}\right.$ size $)+c_{1} \exp \left(-d_{1} \quad \operatorname{size}^{2}\right)-1$ & $\mathrm{a}_{1}=0.791, \mathrm{~b}_{1}=-0.003, \mathrm{c}_{1}=0.789, \mathrm{~d}_{1}=0.216$ \\
\hline $\mathrm{c}_{\text {size }}$ & $1-a_{2} \exp \left(-b_{2}\right.$ size $)-c_{2} \exp \left(-d_{2}\right.$ size $\left.^{2}\right)$ & $\mathrm{a}_{2}=0.479, \mathrm{~b}_{2}=-0.010, \mathrm{c}_{2}=1.290, \mathrm{~d}_{2}=0.176$ \\
\hline
\end{tabular}

$\dagger$ The units of $\mathrm{a}_{1}, \mathrm{c}_{1}, \mathrm{a}_{2}, \mathrm{c}_{2}$ are $\mathrm{ns} / \mathrm{m}$

1)The shower slope falls with age in each shower size bin.

2)The shower slope is higher for a shower of larger shower size but of the same age.

3)The shower slope falls more rapidly with age for a higher shower size bin.

The variation of shower slope with age in each shower size bin is first parametrized by the equation $\mathrm{M}_{\mathrm{age}}^{\text {size }}=\mathrm{m}_{\text {size }} \cdot \mathrm{age}+\mathrm{c}_{\text {size }}$. The parameters $\mathrm{m}_{\text {size }}$ and $\mathrm{c}_{\text {size }}$ calculated for each shower size bin as shown in Fig. 3. The observed variation of $m_{\text {size }}$ and $c_{\text {size }}$ with shower size has also been parametrized by suitable functional forms as listed in Table 1. It is to be noted that the outliers have been eliminated based on the dependence of shower slope on size and age. The percentage of outliers gradually increases with shower size (9.7\%-13.3\%). A study was also done, to observe the variation of $\mathrm{m}_{\text {size }}$ and $\mathrm{c}_{\text {size }}$ with the zenith angle $(\theta)$ for which the data was divided into different $\sec (\theta)$ bins of width 0.1 from 1 to 1.5 . As observed, there is no significant difference $(\lesssim 1 \%)$ in the values of $\mathrm{m}_{\text {size }}$ and $\mathrm{c}_{\text {size }}$ for each zenith angle bin.

\section{The Results on Angular Resolution}

The angular resolution of the GRAPES-3 array was obtained using three methods namely (1)Odd-Even(OE) Method (2)Right Left(RL) Method (3)Simulations

In the odd-even method, the array is first divided into two sub arrays, the first with even numbered and the second with odd numbered detectors, called the odd and even array respectively. The arrival direction of the shower is then determined independently using the two sub arrays. Since these two subarrays have a very substantial spatial overlap, they provide similar estimate of the EAS direction and the measured angular difference between the odd and even directions $\left(\psi_{\text {odd/even }}\right)$ involves the subtraction of two independent reconstructed angles determined by the two subarrays. Therefore, the error in the angular difference between the odd-even subarrays $\left(\sigma_{\text {odd }}\right.$ even $)$ can be written as $\sigma_{\text {odd/even }}=\sqrt{\left(\sigma_{\text {odd/true }}^{2}+\sigma_{\text {even/true }}^{2}\right)}$ where $\sigma_{\text {odd/true }}$ and $\sigma_{\text {even/true }}$ are the angular resolutions of the odd and even array respectively. The odd and even arrays are identical and the number of detectors triggered in both the odd and even array are approximately equal. Therefore $\sigma_{\text {odd/true }} \sim \sigma_{\text {even/true }}$ and the angular resolution of the odd (or even) subarray will be equal to $\frac{\sigma_{\text {odd/even }}}{\sqrt{(2)}}$. The total number of detectors triggered in the full array is double the number in the sub-array and

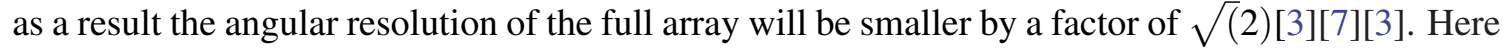
$\sigma_{\text {odd/even }}$ is defined as the FWHM of the $\psi_{\text {odd/even }}$ distribution divided by 2.35 .

From OE method, the angular resolution of the array was obtained to be $1.1^{0}$. The outliers were removed iteratively which led to a net angular resolution of $0.7^{0}$. It should be noted that this method is not sensitive to the systematic tilts in the arrival direction, as the two reconstructed directions are affected in the same way and preferentially tilted in the same direction. Due to this 
reason, the angular resolution obtained through this method sets the lower limit for the angular resolution that is practically obtainable. As expected, the angular resolution decreases with shower size due to the increase in the number of detectors triggered. The angular resolution obtained through OE method for various stages of outlier removal are shown in Figure 4.

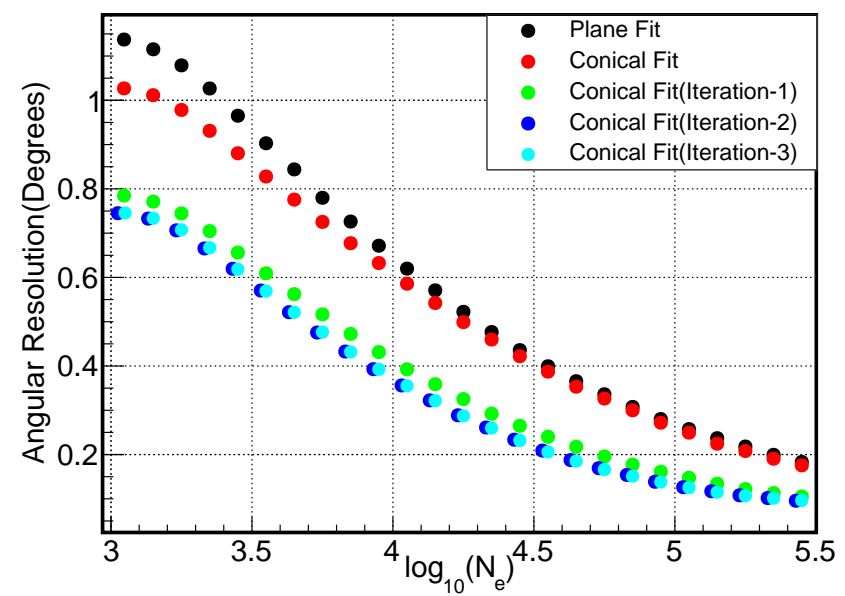

Figure 4: The variation of angular resolution with shower size for various stages of outlier removal for OE method

A more realistic measure of angular resolution can be obtained through the right-left method. In this method, the array is divided by a line joining the shower core with the center of the array, thereby forming two independent subarrays namely the left- and right-half arrays. As in the previous case, the arrival directions are determined independently using the two subarrays and the angular resolution of each subarray is $\frac{\sigma_{\text {right/left }}}{2 \sqrt{(2)}}$. Since angular resolution is inversely proportional to the linear extent of the array, we expect the angular resolution to worsen by a factor of $1 / \sqrt{(2)}$ in the left right case as compared to even-odd case.Therefore correction by an additional factor of $1 / \sqrt{(2)}$ in the left right case as compared to even-odd case. Therefore the angular resolution of the entire array is $\frac{\sigma_{\text {right/left }}}{2 \sqrt{(2)}}[7]$. Since this method is sensitive to the systematic effects arising out of the non-planar shape of the shower front, the initial planar fit to the arrival times yields an angular resolution $\left(1.3^{0}\right)$ which is significantly larger than the angular resolution obtained from odd-even method .The correction for the conical front led to significant improvement in the angualar resolution of the array and further on, the elimination of outliers led to a net angular resolution of $0.8^{0}$. The angular resolution obtained through RL method for various stages of outlier removal are shown in Figure 5. The angular resolution obtained after outlier removal using RL and OE methods are compared in Figure 6(a).The observation of the dependence of shower curvature on age and shower size led to significant improvement in the angular resolution of the array for larger shower size bins as shown in Figure 6(b). For $\log _{10}\left(\mathrm{~N}_{\mathrm{e}}\right)>5$, the angular resoultion improved by a factor of $\sim 2$.

The angular resolution of the GRAPES-3 array was also obtained through simulations. A total of $10^{8}$ CORSIKA generated EAS were reconstructed to obtain their size, core location and arrival direction. The energy deposited in the scintillator slab by the EAS partciles was then estimated from GEANT-4 based detector simulation. The timing response of the scintillator slab was simulated 


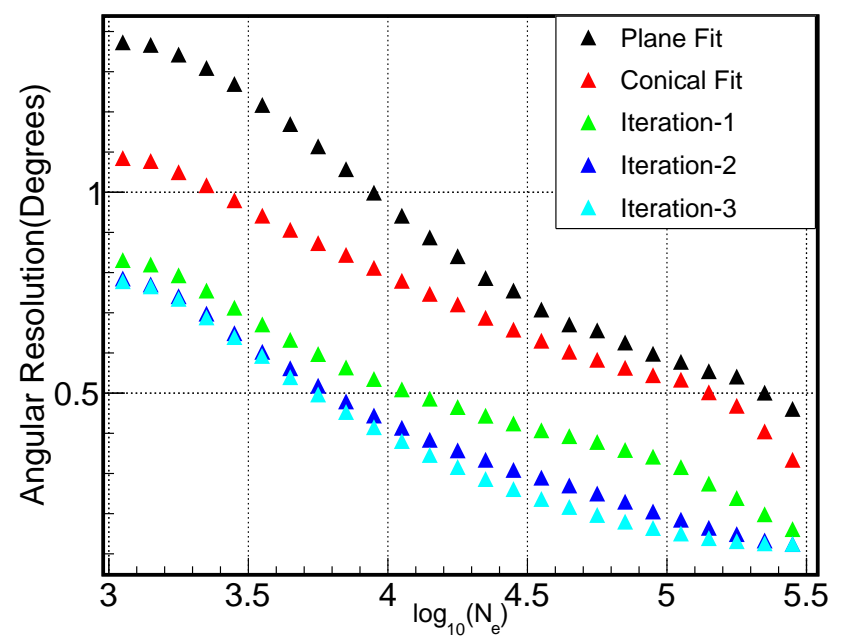

Figure 5: The variation of angular resolution with shower size for various stages of outlier removal for RL method

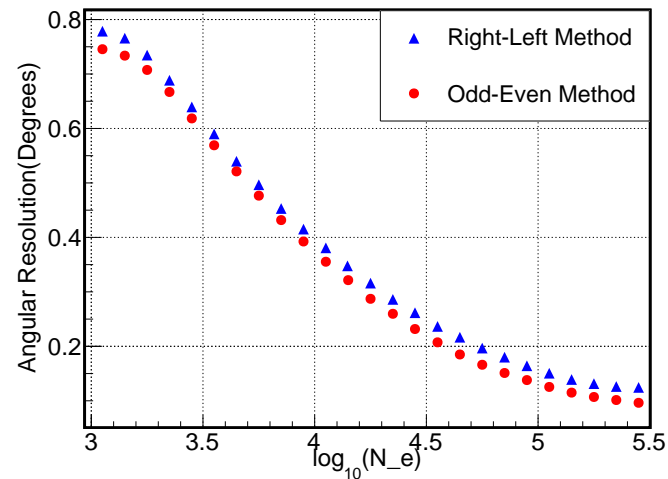

(a) Comparison of OE and RL methods for the third stage of outlier removal

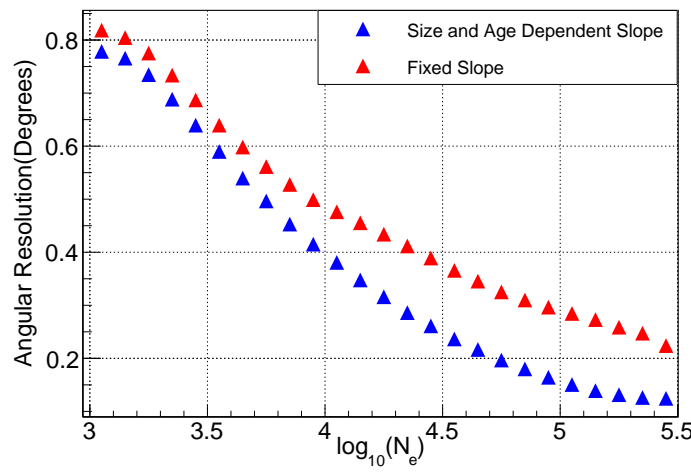

(b) The improvement in the angular resolution of the array on correcting for the shower size and age dependence of the shower slope

Figure 6

based on the observed distribution corresponding to single muons. The angular resolution obtained by comparing the true arrival direction with the reconstructed arrival direction is $0.8^{0}$. The Figure- 7 shows the angular resolution values obtained from simulations as well as data(RL method).

\section{Conclusion}

The sensitivity with which the $\gamma$ ray sources are determined is inversely proportional to the angular resolution of the array. Here the arrival times of particles in an EAS are corrected for the conical shape of the shower front. The slope of the conical shower front was observed to be varying with shower size and age. The correction for the arrival times for this dependence led to significant 


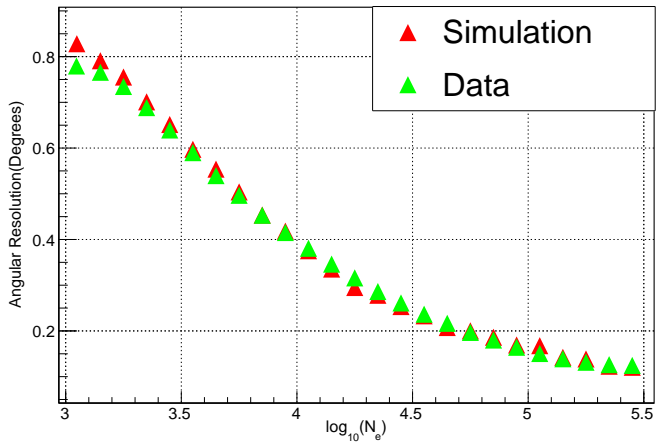

Figure 7: Comparison of angular resolution values obtained from data and simulation

improvement in the angular resolution of the GRAPES-3 array for large shower size bins. From this study the angular resolution of the array is $0.8^{0}$ at $\sim 4 \mathrm{TeV}$. The angular resolution improves to $0.2^{0}$ at $\sim 100 \mathrm{TeV}$ and to $0.1^{\mathrm{O}}$ at $\sim 700 \mathrm{TeV}$.

\section{References}

[1] G. Agnetta, M. Ambrosio, C. Aramo, G. Barbarino, J. Beaman, B. Biondo, D. Campana, O. Catalano, L. Colesanti, G. D'Ali, et al. Time structure of the extensive air shower front. Astroparticle Physics, 6(3-4):301-312, 1997.

[2] T. Antoni, W. Apel, A. Badea, K. Bekk, K. Bernlöhr, H. Blümer, E. Bollmann, H. Bozdog, I. Brancus, A. Chilingarian, et al. Time structure of the extensive air shower muon component measured by the kascade experiment. Astroparticle Physics, 15(2):149-165, 2001.

[3] G. Di Sciascio and E. Rossi. Measurement of the angular resolution of the argo-ybj detector. arXiv preprint arXiv:0710.1945, 2007.

[4] S. Gupta, Y. Aikawa, N. Gopalakrishnan, Y. Hayashi, N. Ikeda, N. Ito, A. Jain, A. John, S. Karthikeyan, S. Kawakami, et al. Grapes3-a high-density air shower array for studies on the structure in the cosmic-ray energy spectrum near the knee. Nuclear Instruments and Methods in Physics Research Section A: Accelerators, Spectrometers, Detectors and Associated Equipment, 540(2-3):311-323, 2005.

[5] H.Tanaka. Energy spectrum and nuclear composition of primary cosmic rays with grapes-3 air shower experiment, 2006.

[6] A. C. Melcarne, L. Perrone, A. Surdo, et al. Time structure of the extensive air shower front with the argo-ybj experiment. Proc. 31st ICRC, 2009.

[7] A. Oshima, S. Dugad, U. Goswami, S. Gupta, Y. Hayashi, N. Ito, A. Iyer, P. Jagadeesan, A. Jain, S. Kawakami, et al. The angular resolution of the grapes-3 array from the shadows of the moon and the sun. Astroparticle Physics, 33(2):97-107, 2010.

[8] R. M. VS and B. V. Sreekantan. Extensive air showers. World Scientific, 1998. 